Abstract PTU-20 Table 1 Accuracy of OD surveillance intervals compared with histopathology-derived intervals

\begin{tabular}{llll}
\hline Group & $\begin{array}{l}\text { Confidence in optical } \\
\text { diagnosis of polyps }<1 \mathrm{~cm}\end{array}$ & Cases & $\begin{array}{l}\text { Accuracy of optical diagnosis } \\
\text { BSG surveillance interval }\end{array}$ \\
\hline A & High confidence only & $47.4 \%$ & $100 \%$ \\
& & $(65 / 137)$ & $(65 / 65)$ \\
B & Mixed (high/low) confidence & $52.6 \%$ & $98.6 \%$ \\
& & $(72 / 137)$ & $(71 / 72)$ \\
\hline
\end{tabular}

surveillance intervals. We analysed the effect of $\mathrm{OD}$ on the accuracy of surveillance interval in a bowel cancer screening setting.

Methods Eight screening colonoscopists used an OD approach for polyps $<1 \mathrm{~cm}$ in 250 patients between Feb-Nov 2020 in the early phase of a prospective feasibility study (DISCARD3). After OD (white light+NBI) all polyps were resected and retrieved for histopathology where possible. Cases with polyps $\geq 1 \mathrm{~cm}$ were excluded.

Cases were divided into 2 groups:

3. Group A: all polyps $<1 \mathrm{~cm}$ and only high confidence OD

- Group B: all polyps $<1 \mathrm{~cm}$ and mixed (high/low) confidence OD

Each patient had an OD surveillance interval assigned and this was compared to histopathology alone.

Results Of 205 patients, 137 were included (68 excluded: 36 had no polyps, 13 had large colorectal cancers, 10 flexible sigmoidoscopies, 7 did not consent, 1 abandoned procedure, 1 missing data). See Table 1.

$65 / 137$ (47.4\%) patients had polyps $<1 \mathrm{~cm}$ and only high confidence OD (Group A). In 65/65 (100\%) cases the OD surveillance interval matched the histology surveillance interval.

$72 / 137$ (52.6\%) patients had polyps $<1 \mathrm{~cm}$ and mixed (high/low) confidence OD (Group B). In 71/72 (98.6\%) cases the OD surveillance interval matched the histology surveillance interval. The one case where surveillance interval changed was a serrated polyp which was found to have dysplasia on histology.

The overall accuracy of OD surveillance intervals compared with histology surveillance intervals was 99.3\% (136/137). There were no unexpected polyp cancer cases.

Conclusions OD of polyps $<1 \mathrm{~cm}$, with a resect and discard strategy, does not appear to adversely affect BSG surveillance intervals across different levels of OD confidence.

\section{PTU-21 SINGLE OPERATOR CHOLANGIOSCOPY - DOES CONSCIOUS SEDATION HAVE A ROLE? A RETROSPECTIVE SINGLE CENTRE EXPERIENCE}

Ann Archer* ${ }^{*}$ KWM Abeysekera, James Hawken, AJ Portal. University Hospitals Bristol and Weston, Bristol, UK

\subsection{6/gutjnl-2021-BSG.94}

Introduction Single operator cholangioscopy (SOC) has an important role in the diagnosis and management of biliary strictures and complex biliary stones. The evidence base is evolving, but with small numbers in reported studies. The majority of published literature discusses SOC under general anaesthetic; typically propofol. We compared successful stone clearance, stricture assessment and safety under conscious sedation and propofol.

Methods A single centre retrospective analysis was performed of consecutive SOCs over 8 years at Bristol Royal Infirmary (tertiary referral centre for SW England and South Wales). Parameters included sedation/propofol usage, stone clearance, electrohydraulic lithotripsy (EHL), histology, final diagnosis and complications.

Results Between Jan 2013 - Nov 2020, 471 SOCs were performed on 352 patients $(175 \mathrm{f}, 177 \mathrm{~m})$; mean age 65.7 years (17-92), referred from 18 centres. 15 SOCs were performed in 2013 compared to 99 in 2019 before restrictions from the COVID19 pandemic.

228 therapeutic SOCs + EHL were performed for stone clearance, $79.8 \% \quad(n=182 / 228) \quad$ under conscious sedation (median fentanyl dose $150 \mathrm{mcg}$; midazolam $7 \mathrm{mg}$; comfort score 2). Complete stone clearance rose from $79.7 \%$ in the conscious sedation group to $89.2 \%$ in with propofol sedation, although the difference was not significant $(p=0.19)$.

243 diagnostic SOCs were performed, $88.9 \%$ under conscious sedation. Similar to therapeutic SOCs, median fentanyl dose $150 \mathrm{mcg}$; midazolam $7 \mathrm{mg}$; and comfort score 2. Overall macroscopic assessment of strictures correlating with malignancy had a sensitivity, specificity and diagnostic accuracy of 90.2\% (95\%CI 82.7-95.2), 92.6\% (95\%CI 86.3-96.5) and $91.5 \%(87.0-94.8 \%)$ respectively. Diagnostic accuracy with macroscopic assessment was comparable between patients receiving conscious sedation $(91.4 \%$; 95\%CI $86.6-94.9)$ vs propofol sedation (92\%; 95\%CI 74.0-99.0). Diagnostic accuracy with histological assessment appeared greater in patients receiving propofol sedation (95\%; 95\%CI 75.1-99.9) compared to conscious sedation $(84.7 \%$; 95\%CI 78.5-89.6)

28 adverse events were recorded (5.9\%), with post ERCP cholangitis $(2.3 \%)$ and pancreatitis $(2.1 \%)$ the commonest causes. Complications were marginally higher in patients receiving propofol sedation $(6.8 \% ; n=5 / 73)$ vs those receiving conscious sedation $(5.8 \% ; \mathrm{n}=23 / 398)$ but the difference was not significant $(\mathrm{p}=0.72)$.

Conclusions This is the largest single centre retrospective analysis of SOCs. Successful stone clearance is similar to results found in literature from smaller studies. There was a trend to greater successful stone clearance with propofol. Conscious sedation has previously been described as a risk factor for inadequate visualisation, but we found very similar high levels of diagnostic accuracy in both groups. Both were generally well tolerated with low rates of adverse events.

\section{PTU-22 IDENTIFYING AND IMPROVING RATES OF POST- COLONOSCOPY COLORECTAL CANCER (PCCRC)}

Clare Blackwell*, Grace Blackmun, Robert Hill, James Shutt, Stephen Bridger. Dorset County Hospital, Bournemouth, UK

\subsection{6/gutjnl-2021-BSG.95}

Introduction Bowel cancer is the fourth commonest cancer in the UK; accounting for over 40,000 new cancer diagnoses and 16,000 deaths per year. ${ }^{1}$ PCCRC (post-colonoscopy colorectal cancer) rate - cancer found within 6 to 36 months after a negative colonoscopy ${ }^{2}-$ is improving. However, there remains a large variation amongst providers, with rates from 3.6- $9.3 \%$. $^{3}$ Early detection leads to improved outcome and 